\title{
Symmetry induced Dynamics in four-dimensional Models deriving from the van der Pol Equation
}

\author{
Ricardo López-Ruiz ${ }^{\dagger} \ddagger$ \\ ${ }^{\dagger}$ Department of Computer Science, Faculty of Sciences, \\ †Instituto de Biocomputación y Física de Sistemas Complejos, \\ Universidad de Zaragoza, 50009 - Zaragoza (Spain)
}

\begin{abstract}
Different models of self-excited oscillators which are four-dimensional extensions of the van der Pol system are reported. Their symmetries are analyzed. Three of them were introduced to model the release of vortices behind circular cylinders with a possible transition from a symmetric to an antisymmetric Bénard-von Karman vortex street. The fourth reported self-excited oscillator is a new model which implements the breaking of the inversion symmetry. It presents the phenomenon of second harmonic generation in a natural way. The parallelism with second harmonic generation in nonlinear optics is discussed. There is also a small region in the parameter space where the dynamics of this system is quasiperiodic or chaotic.
\end{abstract}

Keywords: symmetries, van der Pol-like systems, self-excited oscillators, second harmonic generation, chaotic dynamics

PACS number(s): 05.45.-a, 47.52.+j 


\section{Introduction}

Self-sustained oscillations are found very often in mechanical, electrical, biological, chemical and ecological systems [1]. Nonlinearities are required in order to attain this kind of behavior. The system reaches an stable oscillatory dynamics as a consequence of an internal balance between amplification and dissipation. For instance, the oscillatory patterns in some chemical reactions [2], the beating of the heart [3], the oscillating coexistence between different living being species [4], the Bénard-von Karman vortex street in the wake of a cylinder [5], etc. are examples of continuous and discrete systems modeled as self-excited oscillators.

In this work, five different models of self-excited oscillators with certain similarities are collected. First, the fundamental brick of all of them, the van der Pol equation, is recalled. Second, the two-dimensional van der Pol equation is extended to a four-dimensional model in the complex plane. Two other models deriving from the former complex van der Pol system are revisited. They were investigated as models for the shedding of vortex structures in the flow behind a cylinder. In a next section, a new model of two coupled self-excited oscillators implementing the correct symmetries to get second-harmonic generation is introduced and its qualitative dynamical behavior is sketched. Finally, the conclusions are presented.

\section{Four-dimensional extensions of the van der Pol Model: symmetries and dynamics}

The van der Pol equation, which displays a wide range of behavior from weakly nonlinear to strongly nonlinear relaxation oscillations, is one of the simplest models presenting self-oscillations. It reads:

$$
\ddot{x}-\epsilon\left(1-x^{2}\right) \dot{x}+x=0
$$


where $x(t)$ is recording the time evolution of a harmonic oscillator perturbed by the nonlinear dissipative term $\epsilon\left(1-x^{2}\right) \dot{x}$.

The system (11) presents the following symmetries:

(i) Temporal translation symmetry: if $x(t)$ is a solution of equation (11) then $x(t+\phi)$, with $\phi$ a real constant, is also a solution of system (11) ;

(ii) Spatial inversion symmetry: if $x(t)$ is a solution of the van der Pol equation then $-x(t)$ verifies also the equation (11).

(iii) $\epsilon$-Parameter inversion symmetry: if $x(t)$ is a solution of equation (11) for the constant $\epsilon$ then $-x(-t)$ is also a solution for the parameter $-\epsilon$. Therefore, it is sufficient to find the solutions of equation (11) for $\epsilon>0$. The dynamics for $\epsilon<0$ is derived by applying this symmetry to the latter solutions.

The dynamics of the van der Pol system is quite simple. It presents a fixed point at the origin and a unique periodic orbit for the whole range of the parameter $\epsilon$. For $\epsilon<0$, this limit cycle is unstable, and, for $\epsilon>0$, the limit cycle becomes attractive. Due to the uniqueness of this periodic solution, $\bar{x}(t)$, all the solutions generated from $\bar{x}(t)$ by the symmetries (i) and (ii) fall on the same orbit in the phase plane representation given by the coordinates $(x(t), \dot{x}(t))$. Therefore, there exists a $\bar{\phi}$ satisfying the equation $\bar{x}(t+\bar{\phi})=-\bar{x}(t)$, or equivalently, $\bar{x}(t+2 \bar{\phi})=\bar{x}(t)$. If we call $T$ the period of the limit cycle, that is $\bar{x}(t+T)=\bar{x}(t)$, the symmetries of the equation (11) impose the condition $2 \bar{\phi}=T$. Then, the final dynamical regime verifies $\bar{x}(t+T / 2)=-\bar{x}(t)$. Let us remark that this property is a consequence of the symmetries and the uniqueness of the stable periodic solution of system (11).

The natural extension of the van der Pol equation to a four-dimensional model [5] can be achieved by introducing in the equation (11) the complex variable $z=x_{1}+i x_{2}$. Thus we obtain the complex differential equation

$$
\ddot{z}-\epsilon\left(1-|z|^{2}\right) \dot{z}+z=0
$$

where the real variables $\left(x_{1}, x_{2}\right)$ are the coordinates of two symmetric coupled van der Pol self-excited oscillators which verify: $\ddot{x}_{i}-\epsilon\left(1-\left(x_{1}^{2}+x_{2}^{2}\right)\right) \dot{x}_{i}+x_{i}=0$, with 
$i=1,2$.

Equation (2) has the symmetries:

(i) Temporal translation symmetry: $z(t) \rightarrow z(t+\phi)$, with $\phi$ a real constant;

(ii) Phase symmetry: $z(t) \rightarrow e^{i \psi} z(t)$ with $\psi$ real constant. It includes the spatial inversion symmetry: $z(t) \rightarrow-z(t)$ when $\psi=\pi$;

(iii) Conjugation symmetry: $z(t) \rightarrow \bar{z}(t)$ with $\bar{z}(t)=\left(x_{1}(t),-x_{2}(t)\right)$.

(iv) $\epsilon$-Parameter inversion symmetry: $(z(t), \epsilon) \rightarrow(-z(-t),-\epsilon)$.

The dynamics of system (2) is damped to zero when $\epsilon<0$ and gives self-sustained oscillations when $\epsilon>0$. These stable periodic motions present the form $z(t)=$ $e^{i \phi} r(t)$ with $\phi$ being a real constant and $r(t)$ satisfying $\ddot{r}-\epsilon\left(1-r^{2}\right) \dot{r}+r=0$. It can be represented as a straight line through the origin in the plane $\left(x_{1}, x_{2}\right)$ at constant angle $\phi, r(t)$ oscillating along this line. Evidently, this solution verifies $z(t+T / 2)=-z(t)$, with $T$ the period of the oscillation. It is found that the trivial periodic solutions $z=e^{ \pm i t}$ are unstable.

Equation (2) has been used in Ref. [5] as a fundamental brick to build a simple model for the release of vortices behind circular cylinders. The parameter $\epsilon$ would depend on the Reynolds number of the flow: $\epsilon<0$ for $R e<R e_{c}$ and $\epsilon>0$ for $R e>R e_{c}$ where $R e_{c}$ represents the critical Reynolds number for the onset of the vortex shedding [6, 7, 8]. By introducing a phase symmetry breaking term controlled by the small parameter $\beta$,

$$
\ddot{z}-\epsilon\left(1-|z|^{2}\right) \dot{z}+z+i \beta \bar{z}=0,
$$

the dynamical properties of equation (2) are modified. Now there are only two oscillating solutions that asymptotically attract the flow: the symmetric mode $\Theta_{1} \equiv$ $\left(x_{1}=x_{2}\right)$ given by $z_{1}=e^{\pi / 4} r_{1}(t)$ and the antisymmetric mode $\Theta_{2} \equiv\left(x_{1}=-x_{2}\right)$ given by $z_{2}=e^{-\pi / 4} r_{2}(t)$, where $r_{1}(t)$ and $r_{2}(t)$ verify $\ddot{r}_{i}-\epsilon\left(1-r_{i}^{2}\right) \dot{r}_{i}+\left[1-(-1)^{i} \beta\right] r_{i}=0$ $(i=1,2)$. Both modes have also the property $z_{i}(t+T / 2)=-z_{i}(t), i=1,2$, with $T$ being the period of the motion. If $\beta>0, \Theta_{1}$ is stable and $\Theta_{2}$ unstable, then the oscillators $\left(x_{1}, x_{2}\right)$ are in phase and the representation of a symmetric vortex 
street is obtained [6]. If $\beta<0, \Theta_{1}$ is unstable and $\Theta_{2}$ stable, then the oscillators $\left(x_{1}, x_{2}\right)$ are out of phase and the representation of an antisymmetric vortex street is therefore obtained [7, 8].

A slight modification of the nonlinear coupling term in the dissipative force of equation (3) produces the model:

$$
\begin{aligned}
& \left.\ddot{x}_{1}-\epsilon\left(1-x_{1}^{2}-(1+\gamma) x_{2}^{2}\right)\right) \dot{x_{1}}+x_{1}+\beta x_{2}=0 \\
& \left.\ddot{x}_{2}-\epsilon\left(1-x_{2}^{2}-(1+\gamma) x_{1}^{2}\right)\right) \dot{x_{2}}+x_{2}+\beta x_{1}=0
\end{aligned}
$$

where the parameter $\gamma$ controls the breaking of the conjugation symmetry. The residual symmetries of this system in the plane $\left(x_{1}, x_{2}\right)$ are:

(i) Temporal translation symmetry: $\left(x_{1}(t), x_{2}(t)\right) \rightarrow\left(x_{1}(t+\phi), x_{2}(t+\phi)\right)$, with $\phi$ a real constant;

(ii) Diagonal reflection symmetry: $\left(x_{1}(t), x_{2}(t)\right) \rightarrow\left(x_{2}(t), x_{1}(t)\right)$;

(iii) $\beta$-Parameter inversion symmetry: $\left(x_{1}(t), x_{2}(t), \beta\right) \rightarrow\left(x_{2}(t),-x_{1}(t),-\beta\right)$.

A more general and 'robust' unfolding for the transition between the modes $\Theta_{1}$ and $\Theta_{2}$ is obtained in this new model [5]. Two different scenarios (Scenario I and Scenario II) for this transition are found. The nonlinear transition is performed by the mediation of two intermediary mixed modes: in Scenario I these mixed modes are unstable and in Scenario II they are stable. The Scenario II has been probably confirmed in the experiment performed by Zhang et al. with two flexible filaments interacting and oscillating in a flowing soap [9].

\section{The Model without Inversion Symmetry}

The inversion symmetry is a fundamental property of the solutions of the former models. We introduce here an inversion symmetry breaking term in the equation (2), namely the quadratic term $\beta z^{2}$. We sketch the drastic consequences of this term 
on the dynamical behavior of the system. The new equation is

$$
\ddot{z}-\epsilon\left(1-|z|^{2}\right) \dot{z}+z+\beta z^{2}=0,
$$

where $\beta$ is the coupling constant. The symmetries preserved in this system are:

(i) Temporal translation symmetry: $z(t) \rightarrow z(t+\phi)$, with $\phi$ a real constant;

(ii) Conjugation symmetry: $z(t) \rightarrow \bar{z}(t)$;

(iii) $\beta$-Parameter inversion symmetry: $(z(t), \beta) \rightarrow(-z(t),-\beta)$. Then it is sufficient to study the solutions of equation (5) for $\beta>0$. The dynamics for $\beta<0$ is derived by applying this symmetry to the solutions found for $\beta$ positive.

If we expand the equation (5) in the real variables $\left(x_{1}, x_{2}\right)$, we have:

$$
\begin{aligned}
& \ddot{x}_{1}-\epsilon\left(1-\left(x_{1}^{2}+x_{2}^{2}\right)\right) \dot{x}_{1}+x_{1}+\beta\left(x_{1}^{2}-x_{2}^{2}\right)=0 \\
& \ddot{x}_{2}-\epsilon\left(1-\left(x_{1}^{2}+x_{2}^{2}\right)\right) \dot{x}_{2}+x_{2}+2 \beta x_{1} x_{2}=0 .
\end{aligned}
$$

When $\beta$ is different from zero, a perturbative analysis similar to that performed in Ref. [5] for the model (44) does not give in this case any relevant information. We have performed the numerical integration of these equations and, roughly, two different dynamical regimes are found. The regions where these dynamical behaviors take place are bounded by two curves, $\beta_{1}(\epsilon)$ and $\beta_{2}(\epsilon)$, in the parameter space $(\epsilon, \beta)$, with $\beta_{1}<\beta_{2}$. For instance, when $\epsilon=1, \beta_{1} \simeq 0.9$ and $\beta_{2} \simeq 1.97$, and the general characteristics of these regions are:

(a) Region $I$ : if $0<\beta<\beta_{1}$ or $\beta>\beta_{2}$, a unique stable periodic orbit exists in phase space. It is found that if the coordinate $x_{2}$ oscillates with period $T_{2}=T$ then the coordinate $x_{1}$ oscillates with the half of a period $T_{1}=T / 2$. The system presents in a natural way the phenomenon of second-harmonic generation in this parameter region (Fig. 1(a));

(b) Region II : if $\beta_{1}<\beta<\beta_{2}$, the dynamics of the system displays three different behaviors in the four-dimensional phase space: quasiperiodicity (Fig. 1(b)), periodic windows where the relation $T_{2}=2 T_{1}$ is also verified (Fig. 1(c)), and chaos (Fig. 1(d)). 
The second-harmonic generation phenomenon in Region I is a consequence of two facts: the symmetries of the system and the existence of only one stable periodic orbit. Let $T$ be the period of this orbit, then $\left(x_{1}(t+T), x_{2}(t+T)\right)=\left(x_{1}(t), x_{2}(t)\right)$. Due to the uniqueness of the stable limit cycle, the solutions generated from the time translation and conjugation transformation of this periodic orbit draw the same path on the plane $\left(x_{1}, x_{2}\right)$. There exists therefore a $\bar{\phi}$ verifying that $\left(x_{1}(t+\right.$ $\left.\bar{\phi}), x_{2}(t+\bar{\phi})\right)=\left(x_{1}(t),-x_{2}(t)\right)$. As in the van der Pol system, the only coherent solution for the equation $x_{2}(t+\bar{\phi})=-x_{2}(t)$ is $\bar{\phi}=T / 2$. Then, $x_{2}(t+T / 2)=-x_{2}(t)$ and $x_{1}(t+T / 2)=x_{1}(t)$, that is $T_{2}=2 T_{1}=T$. The oscillator $x_{1}$ performs two oscillations for each oscillation of the oscillator $x_{2}$. The limit cycle on the plane $\left(x_{1}, x_{2}\right)$ presents hence a pair number of lobes in the direction of the $x_{2}$ axis and it shows an eight-folded shape (Fig. 1(a-c)).

Let $z(t, \beta)$ be the unique stable periodic orbit in the Region I. By numerical inspection we find out that $z(t, \beta=0)=z_{0}(t)=\left(0, x_{2,0}(t)\right)$, with $x_{2,0}(t)$ verifying $\ddot{x}_{2,0}-\epsilon\left(1-x_{2,0}^{2}\right) \dot{x}_{2,0}+x_{2,0}=0$. In order to perform an alternative perturbation analysis [10] in the parameter $\beta$, we expand $z(t, \beta)=\left(x_{1}(t, \beta), x_{2}(t, \beta)\right)$ in power series of $\beta$ :

$$
\begin{aligned}
& x_{1}(t, \beta)=\sum_{j=1}^{\infty} \beta^{j} x_{1, j}(t), \\
& x_{2}(t, \beta)=x_{2,0}(t)+\sum_{j=1}^{\infty} \beta^{j} x_{2, j}(t),
\end{aligned}
$$

where $x_{i, j}(t)(i=1,2 ; j=1, \ldots, \infty)$ is calculated by an iterative process as a function of $x_{2,0}(t)$. Then, $x_{i, j}(t)=\tilde{x}_{i, j}\left(x_{2,0}(t)\right)$, with $i=1,2$ and $j=1, \ldots, \infty$. The tilde of $\tilde{x}_{i, j}$ indicates the different functional dependence of $x_{i, j}$ on $x_{2,0}(t)$. Thus, we have:

$$
\begin{aligned}
& \tilde{x}_{1}\left(x_{2,0}(t), \beta\right)=\sum_{j=1}^{\infty} \beta^{j} \tilde{x}_{1, j}\left(x_{2,0}(t)\right), \\
& \tilde{x}_{2}\left(x_{2,0}(t), \beta\right)=x_{2,0}(t)+\sum_{j=1}^{\infty} \beta^{j} \tilde{x}_{2, j}\left(x_{2,0}(t)\right) .
\end{aligned}
$$

The $\beta$-parameter inversion symmetry $(z(t), \beta) \rightarrow(-z(t),-\beta)$ implies that the 
relations:

$$
\begin{aligned}
& \tilde{x}_{1}\left(-x_{2,0}(t),-\beta\right)=-\tilde{x}_{1}\left(x_{2,0}(t), \beta\right), \\
& \tilde{x}_{2}\left(-x_{2,0}(t),-\beta\right)=-\tilde{x}_{2}\left(x_{2,0}(t), \beta\right),
\end{aligned}
$$

must be verified by the correct power series expansion. Furthermore, the conjugation symmetry $(z(t), \beta) \rightarrow(\bar{z}(t), \beta)$ imposes the conditions:

$$
\begin{aligned}
& \tilde{x}_{1}\left(-x_{2,0}(t), \beta\right)=\tilde{x}_{1}\left(x_{2,0}(t), \beta\right), \\
& \tilde{x}_{2}\left(-x_{2,0}(t), \beta\right)=-\tilde{x}_{2}\left(x_{2,0}(t), \beta\right) .
\end{aligned}
$$

Retaining the correct terms which verify the parity conditions (12,15), we arrive at the correct power series expansion:

$$
\begin{aligned}
& \tilde{x}_{1}=\sum_{j=1}^{\infty} \beta^{2 j-1} \tilde{x}_{1,2 j-1}, \\
& \tilde{x}_{2}=x_{2,0}+\sum_{j=1}^{\infty} \beta^{2 j} \tilde{x}_{2,2 j},
\end{aligned}
$$

where $\tilde{x}_{2,2 j}$ are odd functions of $x_{20}$ and $\tilde{x}_{1,2 j-1}$ are even functions of $x_{20}$ for all $j$. This last property implies that $x_{1}$ is a function of $x_{2,0}^{2}$. Because $x_{2,0}(t+T / 2)=-x_{2,0}(t)$ is verified we find that $x_{2,0}^{2}(t+T / 2)=x_{2,0}^{2}(t)$. Therefore the frequency associated to the oscillator $x_{1}(t)$ is the doubled frequency of the oscillator $x_{2}(t)$, as it has been shown above by the argument of the symmetries.

As an example, the first terms of the expansion (16][17), $x_{1} \simeq \beta x_{1,1}$ and $x_{2} \simeq$ $x_{2,0}+\beta^{2} x_{2,2}$, satisfy the differential equations:

$$
\begin{aligned}
\ddot{x}_{2,0}-\epsilon\left(1-x_{2,0}^{2}\right) \dot{x}_{2,0}+x_{2,0} & =0 \\
\ddot{x}_{1,1}-\epsilon\left(1-x_{2,0}^{2}\right) \dot{x}_{1,1}+x_{1,1} & =x_{2,0}^{2}, \\
\ddot{x}_{2,2}-\epsilon\left(1-x_{2,0}^{2}\right) \dot{x}_{2,2}+\left[1+\epsilon\left(x_{2,0}^{2}\right)\right] x_{2,2} & =-2 x_{2,0} x_{1,1}-\epsilon \dot{x}_{2,0} x_{1,1}^{2} .
\end{aligned}
$$

We have verified the correctness of this expansion numerically, by recording $x_{2,0}(t)$ from equation (18) and by reinjecting this signal in equations (19,20), thus obtaining $x_{1,1}(t)$ and $x_{2,2}(t)$. The comparison of this approximation with the real dynamics of $x_{1}(t)$ and $x_{2}(t)$ derived from equation (51) presents a good agreement for $\beta$ slightly 
different from zero. Also, the dependence on the parameter $\beta$ of the limit cycle period has been calculated by computational methods for different values of $\epsilon$. The results are shown in Fig. 2.

The region of the parameter space where the system is chaotic is also investigated by computational methods. We apply directly the method of calculating the sensitive dependence on initial conditions for each particular pair $(\epsilon, \beta)$ of parameter values. When the system shows exponential dependence on the initial conditions, that is, when two infinitesimal separated initial conditions become separated of the system size order, then the system is chaotic and the pair of parameters is collected. The location of the chaotic region in the parameter space $(\epsilon, \beta)$ is shown in Fig. 3.

Second-harmonic generation is one the first phenomena studied in nonlinear optics [1]. Its discovery [12] put in evidence the possibility to modify the optical properties of a material system under the action of an applied optical field sufficiently intense (laser). Thus, in that process, a fraction of the power in the incident radiation at frequency $w$ is converted to radiation at the second-harmonic frequency $2 w$ (Fig. 4). One of the causes of this phenomenon is the non-vanishing second-order susceptibility $\chi^{(2)}$ of the crystal on which the laser beam is incident. This possibility is only achieved in crystals which do not display inversion symmetry. Therefore one would be tempted to establish some formal relation between the behavior of the model (5) in the Region I and that optical system (Fig. 4). From a qualitative point of view, this model presents the breaking of the inversion symmetry and the frequency doubling phenomenon in a natural way.

\section{Conclusions}

In the past few years, important geometrical and topological studies have been developed for understanding the periodic orbit organization of low dimensional chaotic attractors 13, 14]. However, we still experience, for instance, some difficulty to get 
a good visual understanding of the entangled dynamics of a particle following a chaotic trajectory in a strange attractor, even if the system is a three dimensional one. Probably, this a consequence of the lack of some future technical development that would improve and would approach better this visual representation more than a consequence of the lack of theoretical tools for its comprehension.

Thus, the study of four-dimensional systems can be a challenge in the next future. In this work, different self-excited oscillators which are four-dimensional extensions of the van der Pol equation have been reported. Symmetries play a determinant role in the dynamics of all of them. In the model (2), the circular symmetry forbids the capability of this system to select a final dynamical state with a fixed symmetry. By introducing a phase-symmetry breaking term, $i \beta \bar{z}$, and depending of the sign of the control parameter $\beta$, the dynamics in the model (3) can settle down on two different oscillation states: an in-phase mode $\Theta_{1}$ and another out-of-phase mode $\Theta_{2}$. Each mode might represent a symmetric and an antisymmetric Bénard-von Karman vortex street, respectively [5]. In the model (4), an additional breaking circular symmetry term in the dissipative force, monitored by the parameter $\gamma$, provokes the existence of two possible scenarios for the nonlinear transition between the two oscillation modes, $\Theta_{1}$ and $\Theta_{2}$.

Let us observe that the final model (5) implements the necessary breaking term of the inversion symmetry, $\beta z^{2}$, in order to obtain second-harmonic generation when two identical self-excited oscillators interact. When $\beta=0$ the self-excited oscillators are uncoupled and they oscillate to the same frequency $w$. It can represent a laser beam before reaching a crystal with non-vanishing second-order susceptibility. When laser beam passes through the crystal a nonlinear interaction takes place with the medium. Then the two self-excited oscillators are coupled $(\beta \neq 0)$. One of them preserves the original frequency $w$ and the other one oscillates to the doubled frequency $2 w$ (Fig. 4). Other more complicated phenomena such as quasiperiodicity and chaos are also found in a small and very localized region of the parameter space of the model (5) (Region II ). In the case of its experimental existence, the fact 
that the two signals become chaotic in this regime as a consequence of the nonlinear coupling caused by the crystal would convert this unseen behavior in a surprising phenomenon. 


\section{References}

[1] A.A. Andronov, A.A. Vitt and S.E. Khaikin, Theory of oscillators, Dover, New York (1989).

[2] Y. Kuramoto, Chemical oscillations, waves and turbulence, Springer-Verlag, Berlin (1984).

[3] A.T. Winfree, The timing of biological clocks, Scientific American Library, W.H. Freeman Co. (1986).

[4] R.M. May, "Simple mathematical models with very complicated dynamics", Nature, 261, 459-467 (1976).

[5] R. López-Ruiz, and Y. Pomeau, "Transition between two oscillation modes", Phys. Rev. E 55, R3820-R3823 (1997).

[6] T. Frisch, Y. Pomeau and S. Rica, "Transition to dissipation in a model of superflow", Phys. Rev. Lett. 69, 1644-1647 (1992).

[7] H. Bénard, "Formation de centres de giration a l'arrière d'un obstacle en mouvement", C. R. Acad. Sci. Paris 147, 839 (1908).

[8] Th. von Kármán, "Über den Mechanismus des Flüssigkeits und Luftwiderstandes", Gött. Nachr. Math.-Phys. Klasse 509 (1911).

[9] J. Zhang, S. Childress, A. Libchaber and M. Shelley, "Flexible filaments in a flowing soap film as a model for one-dimensional flags in a two-dimensional wind", Nature 408, 835-839 (2000).

[10] E.J. Hinch, Perturbation Methods, Cambridge University Press, Cambridge (1991).

[11] O. Svelto, Principles of Lasers, Plenum Press, New York (1989).

[12] P.A. Franken, A.E. Hill, C.W. Peters and G. Weinreich, "Generation of optical harmonics", Phys. Rev. Lett. 7, 118-119 (1961). 
[13] H.G. Solari, M.A. Natiello and G.B. Mindlin, Nonlinear Dynamics: a Two Way Trip from Physics to Math, IOP Publishing Ltd, Philadelphia (1996).

[14] R. Gilmore and M. Lefranc, Topology of Chaos, John Wiley \& Sons, Inc., New York (2002). 


\section{Figure Captions}

Fig 1. Projections on the plane $\left(x_{1}, x_{2}\right)$ of different stable attractors of system (5). (a) Projections of the stable limit cycle for $\epsilon=1$ and $\beta=0.15$. The biggest orbit represents the projection on the plane $\left(x_{2}, \dot{x}_{2}\right)$. The smallest one is the projection on the plane $\left(x_{1}, \dot{x}_{1}\right)$. The other eight-shaped orbit is the projection on the plane $\left(x_{1}, x_{2}\right)$. (b) Projection of the quasiperiodic attractor for $\epsilon=1$ and $\beta=1$. (c) Projection of a stable orbit in a periodic window for $\epsilon=1$ and $\beta=1.2$. Also, in this case, $T_{2}=2 T_{1}$. (d) Projection of the chaotic attractor for $\epsilon=1$ and $\beta=1.6$.

Fig 2. (a) Plot of the normalised period, $T / 2 \pi$, of the limit cycle of the van der Pol oscillator as a function of the nonlinear friction strength given by the parameter $\epsilon$. (b) Plot of the normalised period, $T / 2 \pi$, of the limit cycle of system (5) as a function of the coupling parameter $\beta$ for $\epsilon=0.5$ (triangles), $\epsilon=1$ (circles), $\epsilon=1.5$ (diamonds) and $\epsilon=2$ (squares).

Fig 3. Values of the parameters $(\epsilon, \beta)$ where the system (5) is chaotic. The sensitive dependence on initial conditions has been verified by computational methods.

Fig 4. Schematic representation of the second-harmonic generation phenomenon in nonlinear optics. 